\title{
Visual Three-Dimensional Reconstruction of Aortic Dissection Based on Medical CT Images
}

\author{
Xiaojie Duan, ${ }^{1}$ Dandan Chen, ${ }^{1}$ Jianming Wang, ${ }^{1}$ Meichen Shi, ${ }^{1}$ Qingliang Chen, ${ }^{2}$ He Zhao, \\ Ruixue Zuo, ${ }^{1}$ Xiuyan $\mathrm{Li}^{1}{ }^{1}$ and Qi Wang ${ }^{1}$ \\ ${ }^{1}$ Tianjin Key Laboratory of Optoelectronic Detection Technology and Systems, School of Electronics and Information Engineering, \\ Tianjin Polytechnic University, Tianjin 300387, China \\ ${ }^{2}$ Tianjin Chest Hospital, Tianjin 300000, China
}

Correspondence should be addressed to Jianming Wang; wangjianming@tjpu.edu.cn

Received 27 February 2017; Revised 7 May 2017; Accepted 7 June 2017; Published 19 July 2017

Academic Editor: Zhijun Fang

Copyright (C) 2017 Xiaojie Duan et al. This is an open access article distributed under the Creative Commons Attribution License, which permits unrestricted use, distribution, and reproduction in any medium, provided the original work is properly cited.

\begin{abstract}
With the rapid development of CT technology, especially the higher resolution of CT machine and a sharp increase in the amount of slices, to extract and three-dimensionally display aortic dissection from the huge medical image data became a challenging task. In this paper, active shape model combined with spatial continuity was adopted to realize automatic reconstruction of aortic dissection. First, we marked aortic feature points from big data sample library and registered training samples to build a statistical model. Meanwhile, gray vectors were sampled by utilizing square matrix, which set the landmarks as the center. Posture parameters of the initial shape were automatically adjusted by the method of spatial continuity between CT sequences. The contrast experiment proved that the proposed algorithm could realize accurate aorta segmentation without selecting the interested region, and it had higher accuracy than GVF snake algorithm (93.29\% versus $87.54 \%$ on aortic arch, $94.30 \%$ versus $89.25 \%$ on descending aorta). Aortic dissection membrane was extracted via Hessian matrix and Bayesian theory. Finally, the three-dimensional visualization of the aortic dissection was completed by volume rendering based on the ray casting method to assist the doctors in clinical diagnosis, which contributed to improving the success rate of the operations.
\end{abstract}

\section{Introduction}

Aortic dissection $(\mathrm{AD})$ is a cardiovascular disease that is a dangerous threat to human health, which can quickly lead to death [1]. The main reason for this disease is that tissue weakness and high blood pressure lead to one or more aortic tissues perforation(s), blood flow along the intimae resulting in two separate blood flow channels: the true lumen (the primary aorta bed of blood flow) and the false lumen (a channel entirely within the media which appears during an aortic dissection) [2], as shown in Figure 1.

At present, the main separation therapy of aortic dissection is the lumen isolation technique requiring that the clinicians can clearly know the crevasse position, range, quantity, severity, and so on before surgery. In order to improve positive rate of aortic dissection, realize automation guidance to interventional treatment, and achieve precise surgery or postoperative evaluation, the aortic dissection 3D reconstruction system is indispensable. The key technology is to achieve aortic segmentation. Threshold-based methods of image segmentation are challenged by intensity gradients within the image volume [3].

And edge detection methods are challenged by poor contrast in the medical images. Methods based on specific theory introduce mathematical models into image segmentation areas, and active shape model (ASM) belongs to the strategy of "top-down"; it combines the prior knowledge of top floor with information at the bottom of the image characteristics and is able to achieve accurate segmentation of complex medical images [4]. In three-dimensional space, there is continuity between CT image sequences; the thicker the slice is, the robuster the continuity is kept between CT slices. Based on the above methods, this paper adopts the method that combines active shape model with spatial continuity to extract aorta area quickly and accurately, which eliminates 


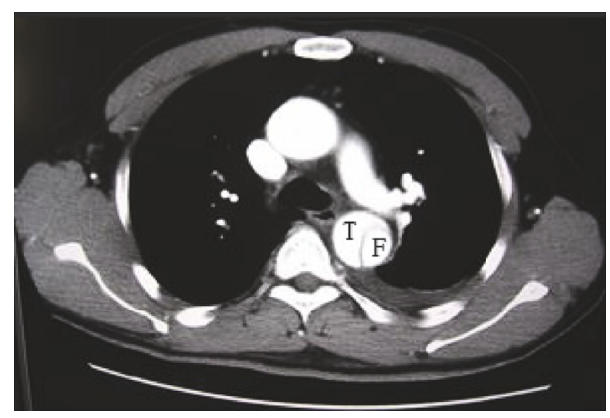

FIgURE 1: The true and the false lumen.

the interference of other organs by shape constraints in the process of extracting the aorta. Then extract aortic dissection membrane using Hessian matrix. And finally, we make use of volume rendering based on ray casting algorithm to perform three-dimensional reconstruction of the aortic dissection. Moreover, we set the transparency and colors of aortic dissection 3D model to make aortic dissection more intuitive; the location and the size of the intimae crevasse can be easily obtained.

\section{Principle of System}

The principle framework of the proposed three-dimensional reconstruction system is shown in Figure 2, via segmentation, extraction, and reconstruction of aortic dissection patients' CT images, to realize three-dimensional visualization of aortic dissection. Firstly, we preprocess the original CT images in order to eliminate noise and adjust the images' brightness for subsequent processing. Secondly, the active shape model is used to segment the aorta region, combined with the spatial continuity of the huge number of CT image sequences, automatically adjusting the posture parameters of the initial shape of each layer to improve the degree of automation and precision of the segmentation algorithm. Next, use gray gradient changes between aortic wall and membrane to extract the aortic dissection membrane by Hessian matrix and continuity a priori model. Finally, three-dimensionally reconstruct the aorta structure by volume rendering and set the transparency and colors of the aortic dissection threedimensional model to make the interval more intuitive and clear. Ultimately, the system can provide help for clinical diagnosis and measures.

\section{Implementation Method}

3.1. Aorta Segmentation. Due to the complexity of the threedimensional structure of the aorta, especially the aortic arch and descending aorta shape big differences, we need to set up aortic arch and descending aorta training set, respectively. In this paper, the training set of the aortic arch and descending aorta are composed of a quantity of samples extracted by equal interval from the aortic dissection patients' CT sequence which contain all the changes in the shape. The two training sets can reflect all the patterns of shape change. We select multiple typical aortic dissection patients' CT images from the Tianjin chest hospital to build the aortic arch and descending aorta model library; different patients have different aortic CT sequence numbers, in the range of about $700 \sim 1000$ on average. In order to model a shape, we represent it by a set of landmark points. This must be done for each shape in the training set and must be done correctly and accurately [5]. The number of feature points on each CT image must be consistent. The labeling is important, and each label represents a particular part of the aorta or its boundary $[6,7]$. In general, we choose points marking parts of the object with particular application-dependent significance, points marking application-dependent things, and other points that can be interpolated from points of types above [8]. Aortic arch and descending aorta landmark points obtained by the expert are shown in Figure 3, and after marking feature points in each CT image we pick up the aortic arch and descending aorta training sample sets are derived, respectively.

According to landmark points, we build aorta big data model library. There is a big deviation in shape and position of different samples, so before modeling, we need to normalized registration shape vectors of two training sets, so as to realize the model building of the aorta [9]. Firstly, select one CT image, respectively, as a benchmark sample from two groups of big data training set, and then scale, rotate, and translate other samples in the library to align them with the benchmark sample; while all the differences between those samples and the reference sample are less than the setting threshold, registration is completed. Registration results of two groups of training set, respectively, are shown in Figure 4, and the horizontal ordinate represents the pixel coordinates in CT image.

Aortic model building is a high-dimensional data processing. In order to simplify calculation process, this paper applies Principal Component Analysis (PCA) approach to reduce dimensionality of the training set which involves all shape vectors to determine the main components [10]. The mean shape vector of the aortic training sample set is calculated by

$$
\begin{aligned}
\bar{X} & =\frac{1}{N} \sum_{i=1}^{N} X_{i}, \\
X_{i} & =\left(x_{i 1}, y_{i 1}, x_{i 2}, y_{i 2}, \ldots, x_{i n}, y_{i n}\right)^{T},
\end{aligned}
$$

where $N$ is the number of the samples of the training set; $X_{i}$ represents the shape vector by stacking $n$ landmark points; then calculate the covariance matrix $S$ by

$$
S=\frac{1}{N-1} \sum_{i=1}^{N}\left(X_{i}-\bar{X}\right)\left(X_{i}-\bar{X}\right)^{T}
$$

Calculate the eigenvectors $\phi_{i}$ corresponding to the eigenvalues $\lambda_{i}$, arrange the eigenvalues in descending order, and choose $k$ eigenvectors which are relative to $k$ bigger eigenvalues, several changing patterns that describe the observed 


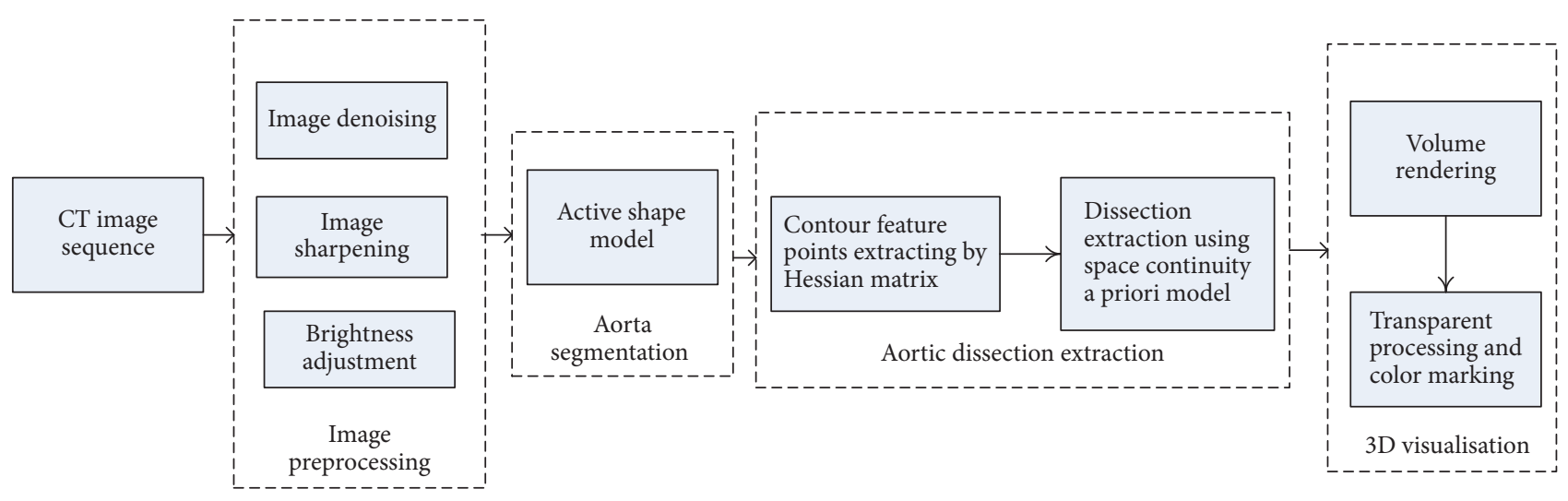

Figure 2: The framework of the three-dimensional reconstruction system.

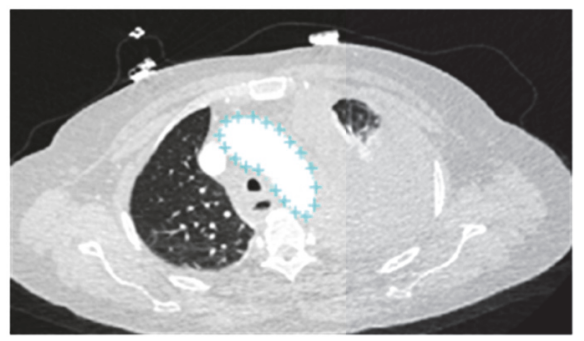

(a) Aortic arch landmark points

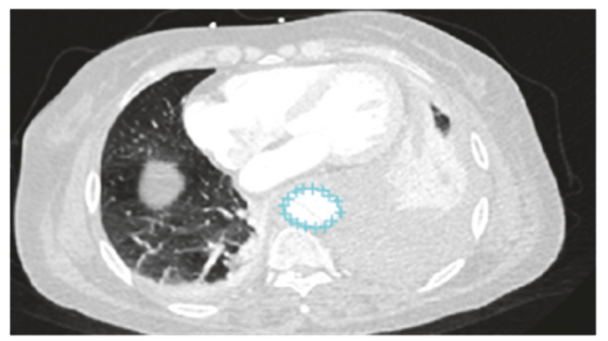

(b) Descending aorta landmark points

FIGURE 3: Landmark points marking.

variation of the training set; at the same time, $k$ eigenvalues need to satisfy the formula as follows:

$$
\sum_{i=1}^{k} \lambda_{i} \geq \rho \sum_{i=1}^{2 n} \lambda_{i}
$$

where $\rho=0.72$ [11]; the search accuracy can reach the highest by the principal components of $72 \%$, and for the general value of $98 \%$, too many constraints will appear when searching. Ultimately, the shape model can be approximated by

$$
X=\bar{X}+\Phi B
$$

where $\Phi$ is the first $k$ eigenvectors, $\Phi=\left(\phi_{1}, \phi_{2}, \ldots, \phi_{k}\right)$, and $B$ is the projection coefficient on the principal component of the shape vector which is calculated as $B=\left(b_{1}, b_{2}, \ldots, b_{k}\right)^{T}$; suitable limits of $b_{i}$ are typically determined in the range of $\left(-3 \sqrt{ } \lambda_{i}, 3 \sqrt{ } \lambda_{i}\right)$, to make sure the active shape model changes within a small range.

The gray-scale texture model is established to match and search target contour at the time that the shape statistical model is built. In this paper, the gray-scale texture model for each landmark is carried out by putting the point as the center of the square of gray sampling, avoiding the traditional method using only the vertical direction information, leading to incomplete search and error convergence. Each landmark gray vector $g_{j i}$ mean and covariance of vector in the training sample can be approximated by

$$
\begin{aligned}
& \bar{g}_{j}=\frac{1}{N} \sum_{i=1}^{N} g_{j i}, \quad j=1,2, \ldots, n, \\
& S_{j}=\frac{1}{N} \sum_{i=1}^{N}\left(g_{j i}-\bar{g}_{j}\right)\left(g_{j i}-\bar{g}_{j}\right)^{T}, \quad j=1,2, \ldots, n .
\end{aligned}
$$

We minimize the Mahalanobis distance between a new profile and the model in the subsequent matching search process as a standard; the matching function is expressed as

$$
f\left(G_{j}\right)=\left(G_{j}-\bar{g}_{j}\right)^{T} S_{j}^{-1}\left(G_{j}-\bar{g}_{j}\right),
$$

where $G_{j}$ is the gray vector of the $j$ th feature point of the matching image; we get the best matching point when the matching function takes the minimum, and when we find all the matching points, the new profile is obtained.

In order to improve the efficiency and robustness of the model, multiresolution search strategy is adopted [12].1 mm thickness at the time of CT scanning is used; therefore a complete set of aorta CT image sequences can be seen continuous, and based on CT image sequence space continuity, by adjusting the initial shape parameters to make the contour close to the target area, the target contour parameters, which are derived from the former image, are used to adjust the current layer, by repeating the above operation between 


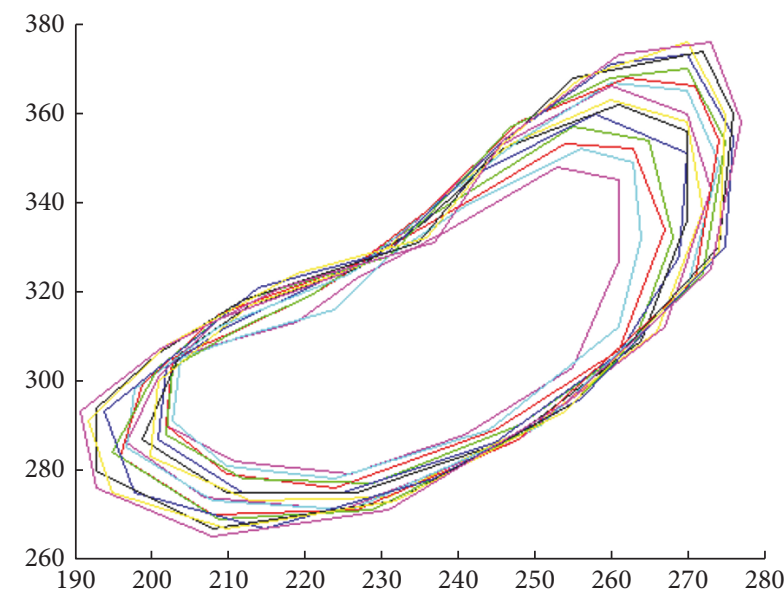

(a) Aortic arch sample set before registration

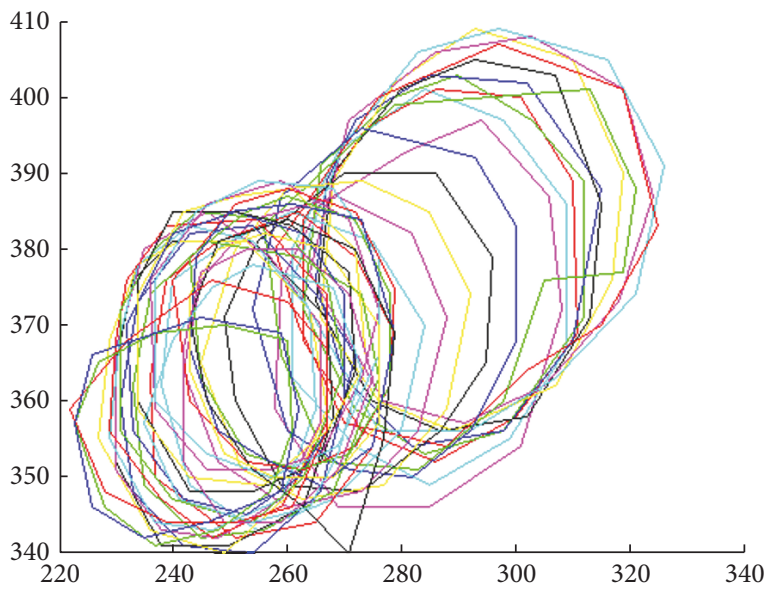

(c) Descending aorta sample set before registration

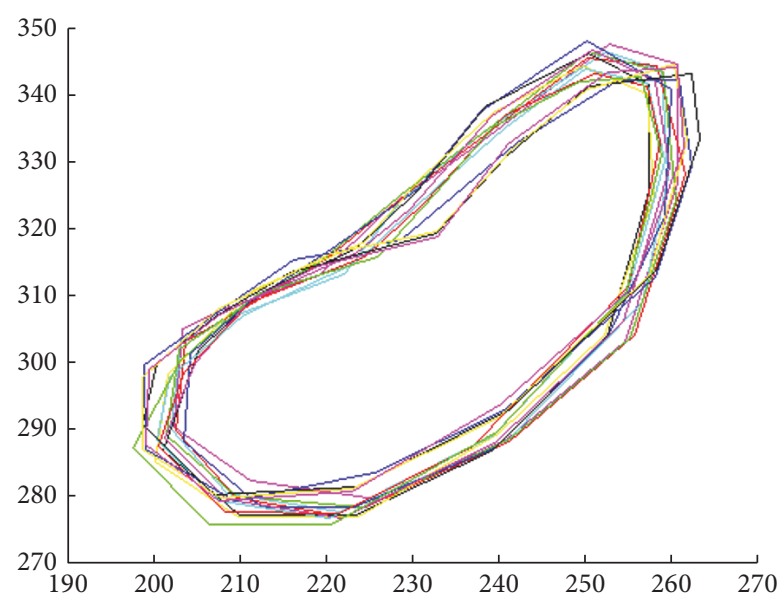

(b) Aortic arch sample set after registration

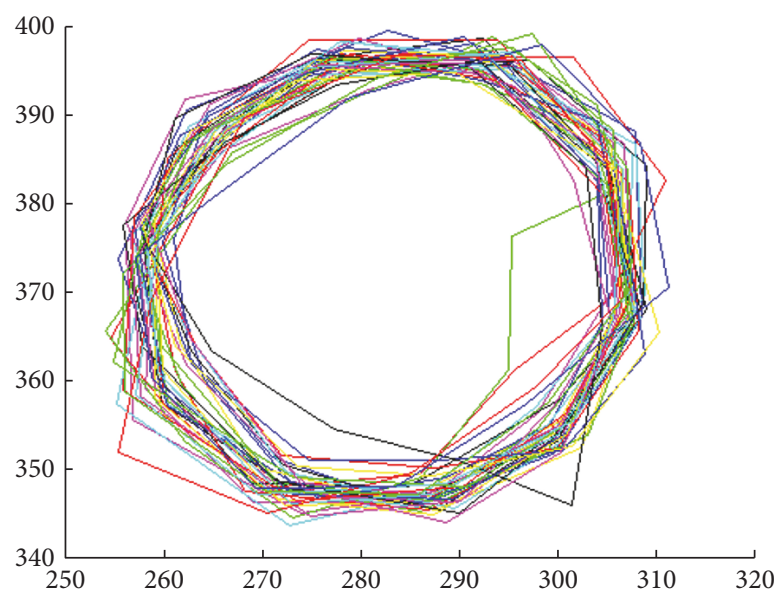

(d) Descending aorta sample set after registration

FIGURE 4: Registration results.

layers. High efficient and accurate segmentation of CT images can be realized; the algorithm is of high degree automation. Figure 5 shows the final segmentation results after iteration convergence.

\subsection{Aortic Dissection Extraction and Three-Dimensional} Reconstruction. In this paper, we propose a detection algorithm combining Hessian matrix and spatial continuity a priori model. The Hessian matrix can be used to extract the pixels on the dissection and aortic boundary [13], the result is shown in Figure 6(a), and then use the Bayesian theory of spatial continuity model to remove other nontarget pixels, that is, only the dissection membrane pixels extracted; the result is shown in Figure 6(b). This algorithm makes full use of the continuity between the CT images of each layer and limits the offset error of the interlayer membrane to a very small range and realizes the accurate extraction of the interlayer membrane.

After the aorta segmentation and dissection membrane extraction are complete, the ray casting is carried out to reconstruct the structure. The ray casting method is a direct volume rendering algorithm based on the image sequence [14]. The specific reconstruction process is as follows.

(1) Read the three-dimensional discrete data field; set different opacity values and color values according to the size of the voxels' pixel values of vertices.

(2) A ray is emitted from each point of the screen based on the direction of the line of sight so that the rays pass through the data field space and $k$ samples are selected equidistantly across all the rays.

(3) The opacity value and the color value of each sampling point are calculated by the trilinear interpolation algorithm using the data values of the eight vertices nearest to the sampling point.

(4) Calculate the opacity and color values for all pixels on the screen based on the cumulative order from front to back.

(5) The opacity value and the color value of each pixel obtained are projected onto the imaging screen to generate the final three-dimensional image. 


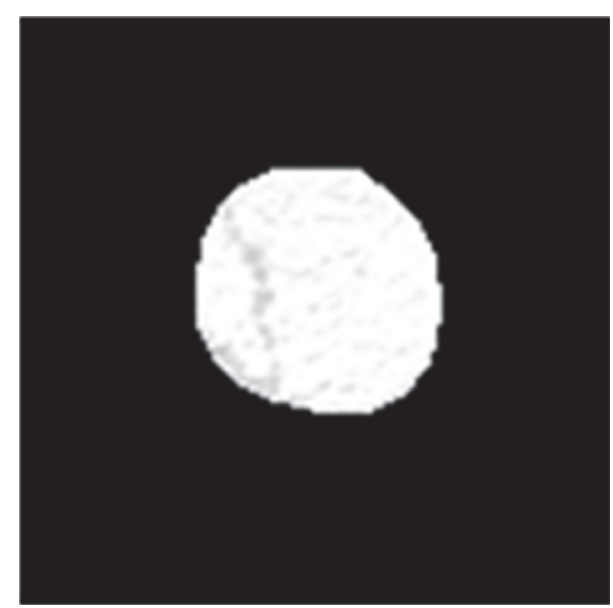

(a) Descending aorta from segmentation

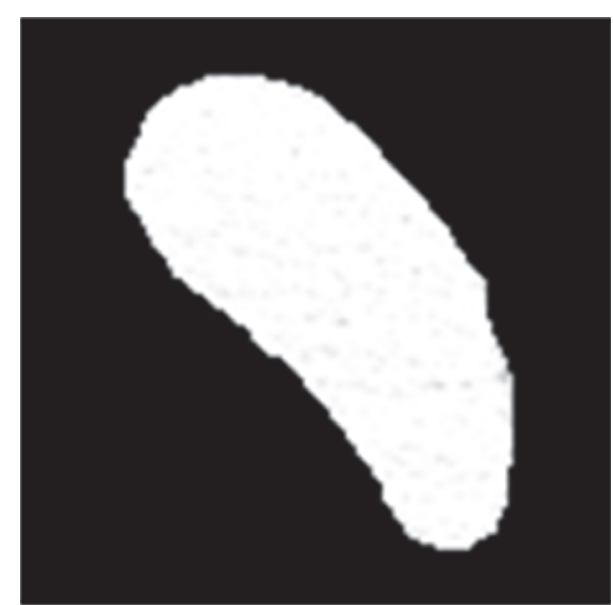

(b) Aortic arch from segmentation

FiguRE 5: Final result after iteration convergence.

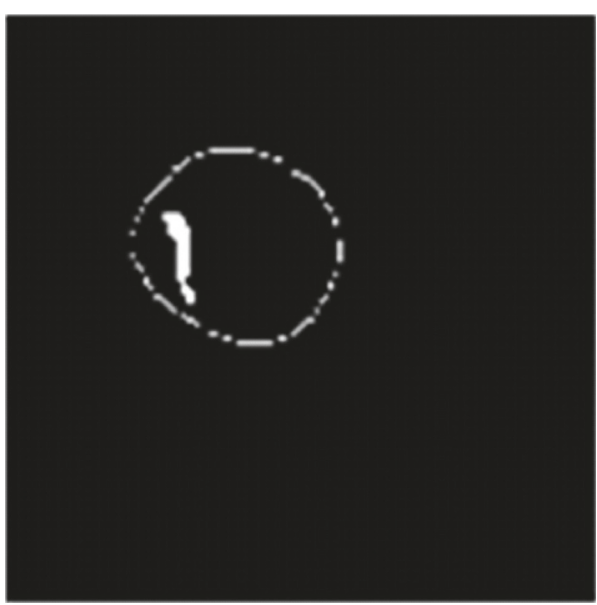

(a) Contour pixel feature points extraction result

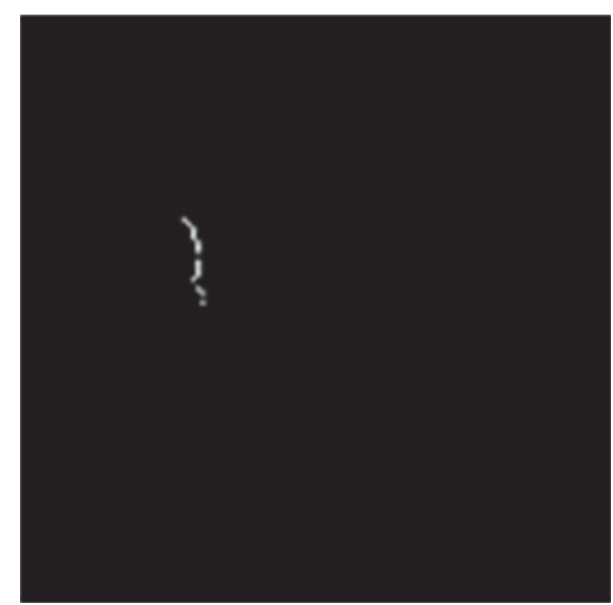

(b) Aortic dissection results

FIGURE 6: Aortic dissection extraction result.

The final 3D reconstruction of a group of aortic region is as shown in Figure 7. By setting the aorta transparency and color, the interlayer is shown in red to give the final reconstruction results, as shown in Figure 8.

\section{Experimental Results and Discussions}

4.1. Experimental Results. Figure 9 shows a three-dimensional model of the aortic dissection of the other two patients reconstructed by the above method. It is clear from the three-dimensional reconstructed images that the dissection membrane occurs in the entire aortic cavity in the first set of models, and the dissection membrane of the second group appears in the aortic arch away from the heart. The results of the two groups intuitively and clearly show the location and extent of the break, so the method provides much more space relationship information of the aortic dissection to the attending physician for the diagnosis of the diseases, surgery, and postoperative evaluation to provide assistance.

4.2. Segmentation Accuracy. In order to verify the algorithm for segmentation of CT image sequence aortic extraction effect, compare the aortic arch and descending aorta extraction results on this proposed method with those based on GVF snake algorithm. To test and verify the reliability of above two algorithms, we compare the extraction results by algorithms above with the manual results by doctor of rich clinical experience and calculate the overlapping rate to test the reliability of the algorithm in this paper; the extraction results by three methods are shown in Figure 10.

We can obtain that, for descending aorta, the segmentation results by both GVF snake model and the proposed segmentation algorithm are close to the real target contour. While for the aortic arch the GVF snake algorithm has certain errors, because of the absence of shape constraints, the 
TABLE 1: Two segmentation algorithms' overlap ratio.

\begin{tabular}{lcccc}
\hline & $\begin{array}{c}\text { GVF snake method } \\
\text { (aortic arch) }\end{array}$ & $\begin{array}{c}\text { Our proposed method } \\
\text { (aortic arch) }\end{array}$ & $\begin{array}{c}\text { GVF snake method } \\
\text { (descending aorta) }\end{array}$ & $\begin{array}{c}\text { Our proposed method } \\
\text { (descending aorta) }\end{array}$ \\
\hline Sample 1 & $84.52 \%$ & $92.32 \%$ & $89.75 \%$ & $95.59 \%$ \\
Sample 2 & $86.51 \%$ & $92.97 \%$ & $88.16 \%$ & $94.98 \%$ \\
Sample 3 & $87.63 \%$ & $93.09 \%$ & $90.03 \%$ & $93.26 \%$ \\
Sample 4 & $89.95 \%$ & $94.65 \%$ & $89.80 \%$ & $93.76 \%$ \\
Sample 5 & $88.02 \%$ & $93.23 \%$ & $88.70 \%$ & $94.07 \%$ \\
Sample 6 & $88.63 \%$ & $93.45 \%$ & $89.04 \%$ & $94.12 \%$ \\
\hline
\end{tabular}
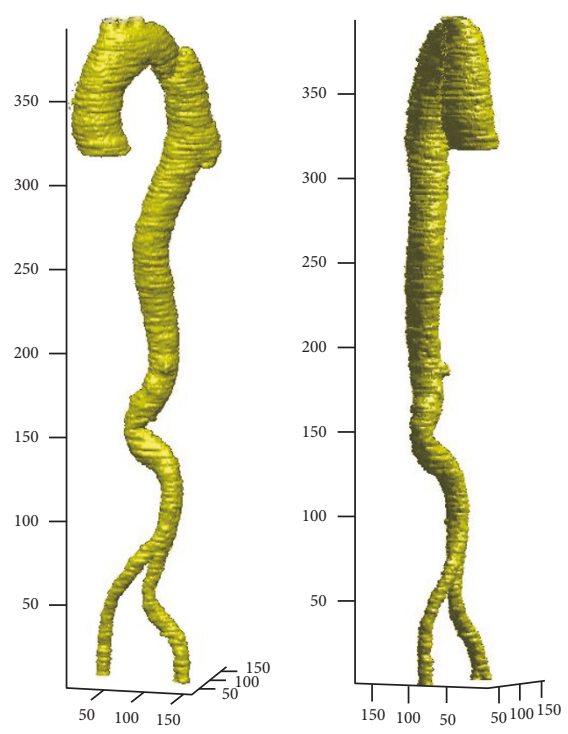

FIGURE 7: 3D visualization of the aorta region.

curve is vulnerable to the interference of other organizations around the outline in the process of evolution, which is shown in Figure 11(a); curve 1 is the initial curve, and curve 2 is the end evolution of the curve. In order to avoid impact from other groups' contour in the curve evolution, the GVF snake model firstly determines the aorta interested area which involves complete aorta area but as far as possible to introduce the interference of other organizations $[15,16]$. Generally speaking, fixed location areas or template matching methods are chosen, which are with low repeatability and adaptability, and the selecting accuracy directly affects the subsequent partitioning extraction results. However we can achieve the aorta automatic segmentation result after directly inputting one CT image with our proposed method. Figure 11(b) shows the iteration results by our proposed method. And this algorithm is of higher degree of automation and stronger robustness than GVF snake model.

In order to quantitatively describe the accuracy of the algorithm in this paper, using the overlap rate as evaluation indexes,

$$
\text { Overlap }=\frac{A_{a b}}{A_{a}+A_{b}-A_{a b}}
$$
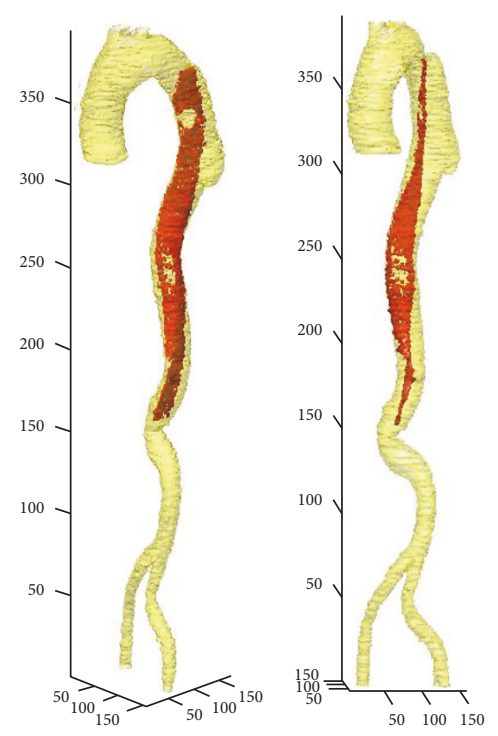

FIGURE 8: 3D visualization of aortic dissection.

$A_{a}$ and $A_{b}$, respectively, represent the proportion of the target area from the two images' segmentation; $A_{a b}$ is the proportion of the target zone overlapping. With thoracic hospital doctors manual segmentation result as the gold standard, our experiment selects large number of images from groups of patients' CT images and then calculates the two algorithms' overlap, respectively, and some samples results of those all are displayed in Table 1.

For extraction accuracy, the proposed method is obviously of higher overlap ratio than the GVF snake method from the table $(93.29 \%$ versus $87.54 \%$ on aortic arch, $94.30 \%$ versus $89.25 \%$ on descending aorta).

\section{Conclusions}

This paper introduces a kind of medical CT image processing method to rapidly and accurately obtain aortic dissection characteristic from huge CT image data and to threedimensionally reconstruct the structure for doctors in clinical diagnosis. The algorithm of this paper has been improved on the basis of the traditional ASM algorithms; not only is the statistical model more accurately constructed and the accuracy of the model matching improved, but also the initialization process combined with the continuity of the 

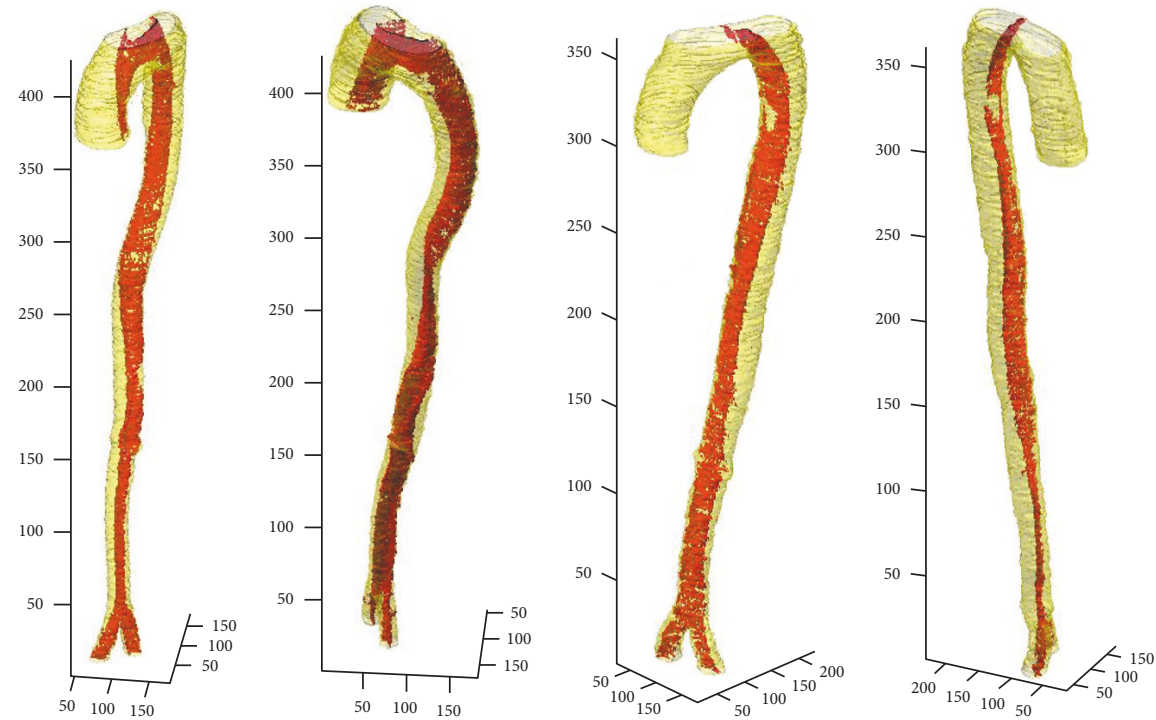

(a) The first patient's aortic dissection display

(b) The second patient's aortic dissection display

FIGURE 9: 3D visualization model of another two patients with aortic dissection.

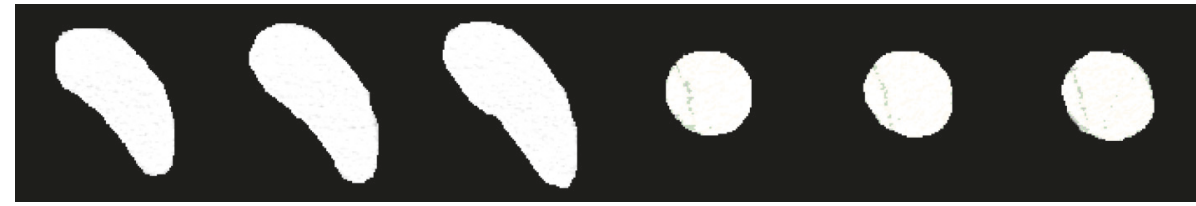

(a) Aortic arch and descending aorta manual segmentation results

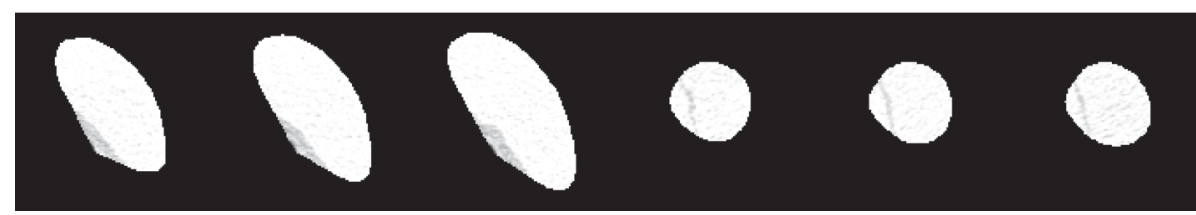

(b) Segmentation results by GVF snake method

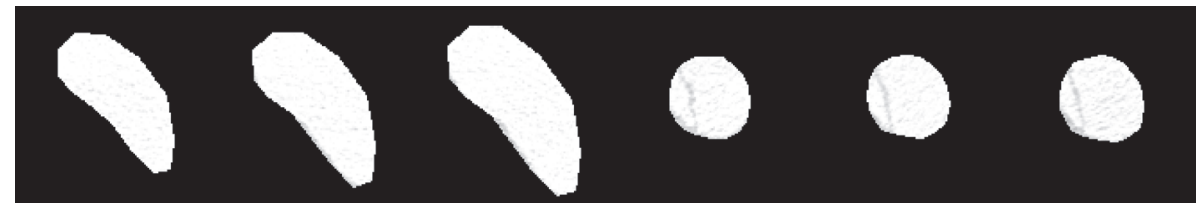

(c) Segmentation results by this proposed method

Figure 10: Aortic arch and descending aorta segmentation results by three methods.

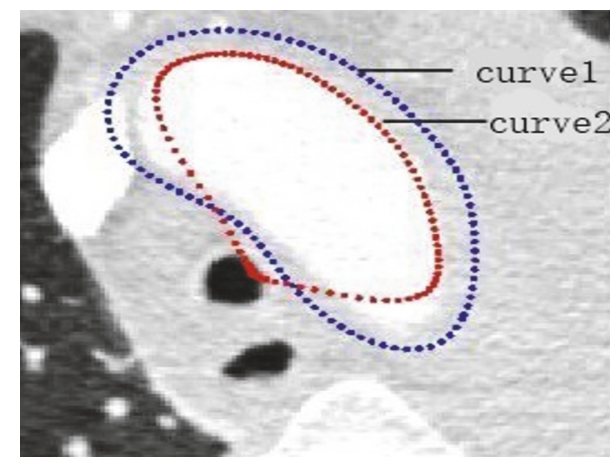

(a) Iteration result based on GVF snake method

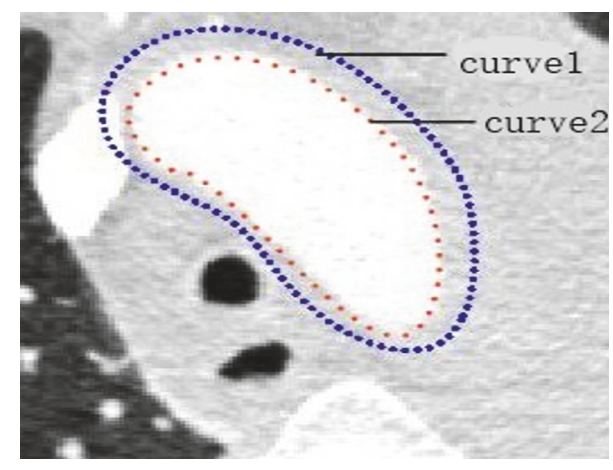

(b) Iteration result based on the proposed method

FIGURE 11: Iteration results based on two methods. 
CT sequence is simplified and the algorithm effectiveness is improved. Compared to GVF snake algorithm, the experiment can be a strong proof that our algorithm can effectively improve the accuracy of aorta segmentation. This method of this paper can effectively make up for the inadequacy of existing hospital equipment function, so that the attending physicians and patients can deeply understand and grasp the state of the illness. At the same time, for other cardiovascular diseases' diagnosis and treatment, this method is of great significance.

\section{Conflicts of Interest}

The authors declare that there are no conflicts of interest regarding the publication of this paper.

\section{Acknowledgments}

This work is supported by the National Natural Science Foundation of China (NSFC) (61405143, 61373104, and 61402330) and Key Projects in the National Science and Technology Pillar Program (2013BAF06B00) under Tianjin Key Laboratory of Optoelectronic Detection Technology and Systems, Tianjin Polytechnic University, China.

\section{References}

[1] N. Fetnaci, P. Lubniewski, B. Miguel, and C. Lohou, "3D segmentation of the true and false lumens on CT aortic dissection images," in Proceedings of the Three-Dimensional Image Processing (3DIP) and Applications 2013, Burlingame, CA, USA, February 2013.

[2] C. Lohou, N. Fetnaci, P. Lubniewski, B. Miguel, P. Chabrot, and L. Sarry, "Intimai flap segmentation on CTA aortic dissection images based on mathematical morphology," in Proceedings of the 2013 6th International Conference on Biomedical Engineering and Informatics, BMEI 2013, pp. 143-147, Hangzhou, China, December 2013.

[3] M. Deeley A, A. Chen, R. Datteri et al., "Comparison of manual and automatic segmentation methods for brain structures in the presence of space-occupying lesions: a multi-expert study," Physics in Medicine and Biology, vol. 56, no. 14, p. 4557, 2011.

[4] T. F. Cootes and C. J. Taylor, "Statistical models of appearance for medical image analysis and computer vision," in Proceedings of the Medical Imaging 2001 Image Processing, pp. 236-248, San Diego, Calif, USA, February 2001.

[5] C. Je, W. Jo, and H.-M. Park, "Mouth map-assisted localisation of ASM lip landmarks," Imaging Science Journal, vol. 64, no. 8, pp. 419-424, 2016.

[6] Z. Zheng, J. Jiong, D. Chunjiang, X. Liu, and J. Yang, "Facial feature localization based on an improved active shape model," Information Sciences, vol. 178, no. 9, pp. 2215-2223, 2008.

[7] G. Gill, M. Toews, and R. R. Beichel, "Robust initialization of active shape models for lung segmentation in CT scans: A feature-based atlas approach," International Journal of Biomedical Imaging, vol. 2014, Article ID 479154, 2014.

[8] T. Cootes, C. Taylor J, and A. Lanitis, "Active shape models: evaluation of a multi-resolution method for improving image search," in Proceedings of the British Machine Vision Conference, pp. 327-336, 2010.
[9] H. Sun, X. Lu, and H. Liu, "A segmentation method of aorta in ct image," Modern Scientific Instruments, no. 02, pp. 45-48, 2013.

[10] J. Wang and C. Shi, "Automatic construction of statistical shape models using deformable simplex meshes with vector field convolution energy," Biomedical Engineering Online, vol. 16, article 49, no. 1, 2017.

[11] S. Kurugol, R. San Jose Estepar, J. Ross, and G. R. Washko, "Aorta segmentation with a 3D level set approach and quantification of aortic calcifications in non-contrast chest CT," in Proceedings of the 34th Annual International Conference of the IEEE Engineering in Medicine and Biology Society (EMBS '12), pp. 2343-2346, IEEE, San Diego, Calif, USA, September 2012.

[12] C.-Y. Tsai, C.-H. Huang, and A.-H. Tsao, "Graphics processing unit-accelerated multi-resolution exhaustive search algorithm for real-time keypoint descriptor matching in high-dimensional spaces," IET Computer Vision, vol. 10, no. 3, pp. 212-219, 2016.

[13] R. Lakemond, C. Fookes, and S. Sridharan, "Affine adaptation of local image features using the Hessian matrix," in Proceedings of the 6th IEEE International Conference on Advanced Video and Signal Based Surveillance, AVSS 2009, pp. 496-501, Genova, Italy, September 2009.

[14] J. Sun, H. Li, P. Gao, and L. Wu, "Research on high efficient ray casting algorithm based on VTK," in Proceedings of the 7th International Conference on Information Technology in Medicine and Education, ITME 2015, pp. 212-214, Huangshan, China, November 2015.

[15] T. Guan, D. Zhou, and Y. Liu, "Accurate segmentation of partially overlapping cervical cells based on dynamic sparse contour searching and GVF snake mode," Journal of Biomedical and Health Informatics, vol. 19, no. 4, pp. 1494-1504, 2015.

[16] F. Zhang, X. Zhang, K. Cao et al., "Contour extraction of gait recognition based on improved GVF Snake model," Computers \& Electrical Engineering, vol. 38, no. 4, pp. 882-890, 2012. 


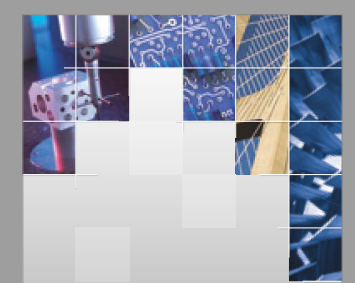

\section{Enfincering}
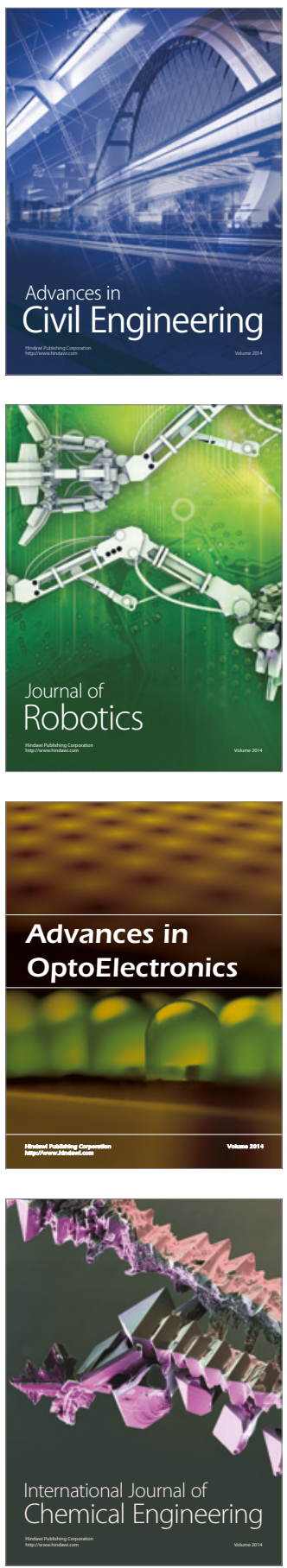

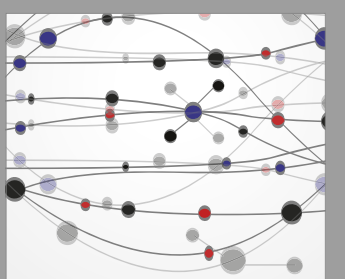

The Scientific World Journal

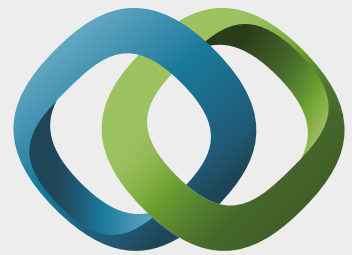

\section{Hindawi}

Submit your manuscripts at

https://www.hindawi.com
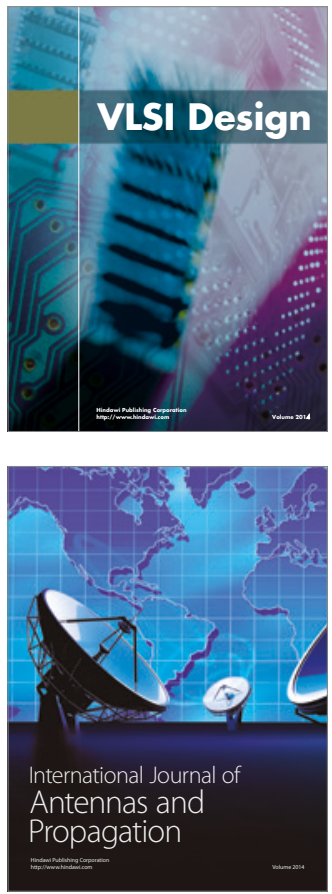

\section{Rotating}

Machinery
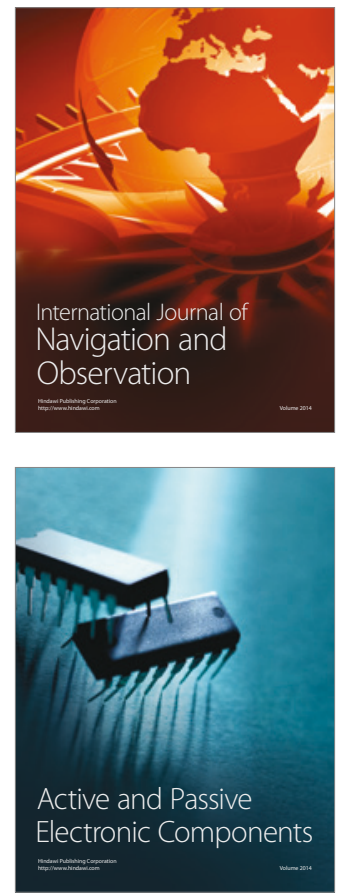
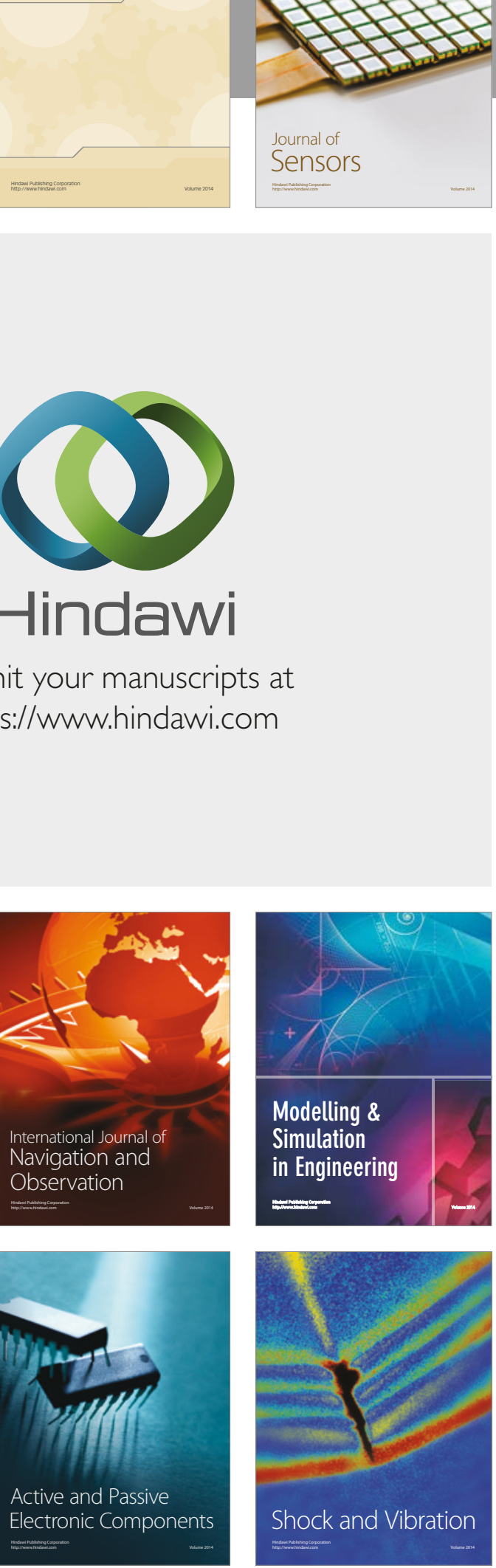
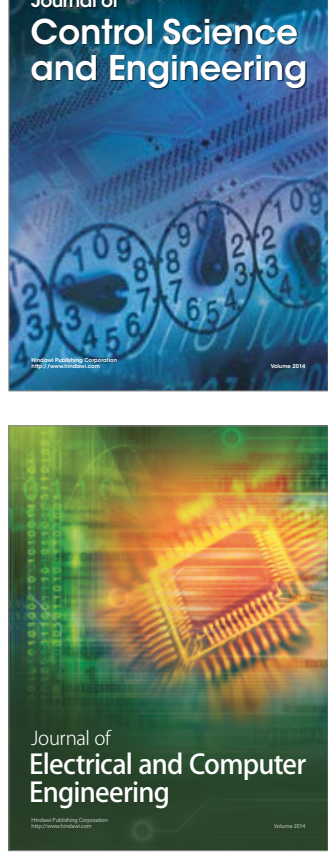

Distributed

Journal of

Control Science

and Engineering
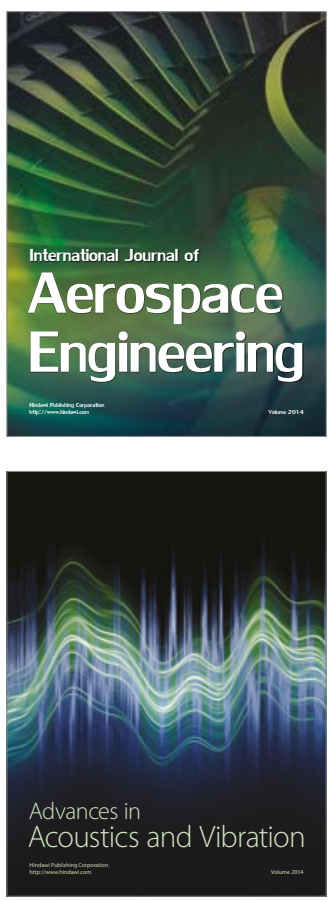

Sensor Networks 